Article

\title{
Drilling Localization and Error Analysis of Radial Horizontal Jet Drilling Based on Magnetic Gradient Tensor
}

\author{
Zhongxiang Liu, Minghui Wei *, Peng Zhang, Yutao Zhang, Tingtao Lu, Rui Xiong and \\ Changchun Qin
}

School of Mechatronic Engineering, Southwest Petroleum University, Chengdu 610500, China; liuzxsc@163.com (Z.L.); 13558800843@163.com (P.Z.); 19802870198@163.com (Y.Z.); lutingtao2020@163.com (T.L.); xir77528@163.com (R.X.); Qinchangchun2020@163.com (C.Q.)

* Correspondence: weiminghui@swpu.edu.cn

Received: 8 August 2020; Accepted: 22 September 2020; Published: 23 September 2020

check for updates

\begin{abstract}
To obtain the real-time drilling trajectory of radial horizontal jet drilling (RHJD), a magnetized nozzle localization method based on magnetic gradient tensor (MGT) is proposed. The MGT system consisting of five tri-axial magnetometers, a tri-axial accelerometer, and a tri-axial gyroscope are installed in the casing. The magnetized nozzle is made of a strong magnetic permanent magnet whose position can be obtained by measuring the MGT generated by itself at the measurement point. The simulation and experiment of the localization method are carried out, which show that the method has high accuracy. When the detection distance is $40 \mathrm{~m}$, the error is only $0.07 \mathrm{~m}$, which meets the requirements of engineering practice. The accuracy of the magnetometer, the baseline distance and the magnetization strength of the nozzle are the main factors affecting the localization error. This localization method can meet the requirements of positioning accuracy in practical engineering, which greatly avoids the drilling failure of RHJD and has a wide application prospect.
\end{abstract}

Keywords: radial horizontal jet drilling; localization; magnetic gradient tensor

\section{Introduction}

Radial horizontal jet drilling (RHJD), that is, using high-pressure water jet to drill multiple horizontal branch boreholes with a length of 20-100 m along the radial direction of the casing at a certain level, or multiple levels, of the oil reservoirs [1-3]. RHJD technology is a low cost, high benefit residual oil recovery technology, which is especially suitable for casing damaged well, low yield well and other old well reconstruction and development of uneconomical thin layer reservoir. In recent years, RHJD has been increasingly applied in oil and gas development. It can improve the pressure distribution of oil and gas reservoirs and has played positive roles in improving the drilling penetration rate and increasing gas and oil recovery [4-6].

Drilling distance is proportional to oil and gas production [7]. Compared with conventional horizontal wells, the curvature radius of the RHJD is smaller, which is generally less than $4 \mathrm{~m}$. As a result, the conventional drilling tools cannot meet the requirements of the process and need to be replaced by a high-pressure hose, with a diameter of only about $30 \mathrm{~mm}$. Due to the insufficient rigidity of the high-pressure hose, it is difficult to maintain a straight state and easy to form a coil in the tubing or spirally drill in the formation. Therefore, it is difficult to obtain the accurate drilling distance and the drilling trajectory is uncontrollable, thereby affecting the drilling effect. In practical engineering, it is necessary to know whether the drilling distance of RHJD reaches $20 \mathrm{~m}$, so as to judge whether the operation is successful. 
To solve the above problems, a localization method based on the magnetic gradient tensor (MGT) is proposed. The magnetized nozzle is regarded as a magnetic dipole, whose position can be obtained by measuring its MGT by the MGT system installed in the casing. The MGT can provide more comprehensive magnetic field information, which is not affected by orientation errors and is insensitive to the noise of spatial orientation and rotation. At the same time, it has a higher spatial resolution and is not easily affected by environmental magnetic interference and geomagnetic diurnal variation [8,9]. In recent years, MGT technology has been continuously developed and improved, which has become a mature technology. It has been widely used in aerial exploration, medical diagnosis and localization of unexploded ordnance, etc. [10-12].

However, considering the interference magnetic field existing in the RHJD project, the localization method based on MGT cannot be directly applied. In this paper, the localization method has been improved so that it can eliminate the interference magnetic field. At the same time, a mathematical model is established according to the engineering requirements of RHJD and the localization algorithm. Then the influence of the distance between source and MGT system, the strength of the equivalent magnetic moment, the accuracy of the magnetometer and baseline distance on positioning error are analyzed, and the MGT system is optimized based on the simulation results. Finally, the effectiveness of the method is verified by the experiments.

The combination of MGT and RHJD can locate the magnetized nozzle in real time and make the drilling trajectory visible. Ineffective drilling can be avoided, which greatly improves the drilling efficiency. Meanwhile, the method is convenient and economical.

\section{Methods}

The schematic diagram of the method is shown in Figure 1. The magnetized nozzle is made of a strong magnetic permanent magnet, and the MGT system is installed in the casing. The MGT system is used to measure the MGT information generated by the magnetized nozzle at the measuring point, and then a difference calculation is carried out to eliminate the effects of the environmental magnetic field. At last, the position of the magnetized nozzle, relative to the MGT system will be solved.

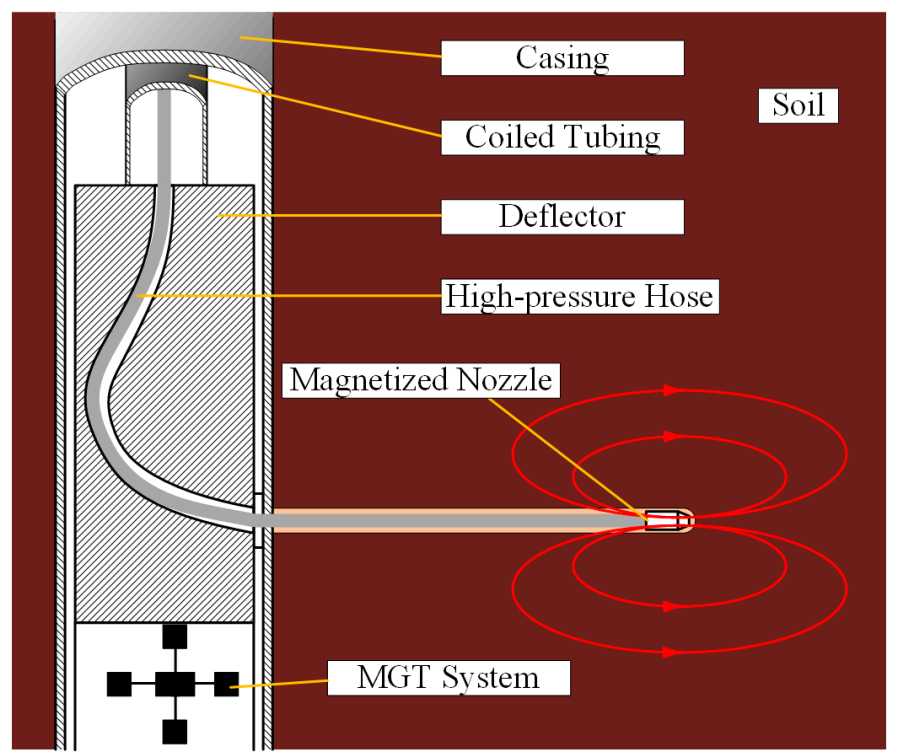

Figure 1. The schematic diagram of the method. MGT: magnetic gradient tensor. 


\subsection{Magnetic Gradient Tensor}

MGT $G$ expresses the spacial rate of change of the magnetic field $\left(B_{x}, B_{y}, B_{z}\right)$ in three orthogonal directions $(x, y, z)$. There are nine elements in $G$ and its expression is as follows:

$$
G=\left[\begin{array}{lll}
\partial B_{x} / \partial x & \partial B_{x} / \partial y & \partial B_{x} / \partial z \\
\partial B_{y} / \partial x & \partial B_{y} / \partial y & \partial B_{y} / \partial z \\
\partial B_{z} / \partial x & \partial B_{z} / \partial y & \partial B_{z} / \partial z
\end{array}\right]
$$

In passive space, the divergence and curl of the magnetic field are equal to zero,

$$
\left\{\begin{array}{c}
\nabla \cdot \mathbf{B}=B_{x x}+B_{y y}+B_{z z}=0 \\
\nabla \times \mathbf{B}=0 \rightarrow B_{x y}=B_{y x}, B_{x z}=B_{z x}, B_{y z}=B_{z y}
\end{array} .\right.
$$

According to Equations (1) and (2), only five of the nine tensor components in $G$ are independent.

\subsection{Localization Algorithm}

If the distance between the detection point and the magnetic source is greater than 2.5 times the size of the source, the magnetic source can be regarded as a magnetic dipole [13].

In cartesian coordinates, the magnetic field vector at $\mathbf{r}$ relative to the magnetic dipole is [14]:

$$
\mathbf{B}=\frac{\mu_{0}}{4 \pi}\left[\frac{3(\mathbf{m} \cdot \mathbf{n}) \mathbf{n}-\mathbf{m}}{r^{3}}\right]
$$

where $\mu_{0}$ is the permeability of vacuum. $r=|\mathbf{r}|$ is the distance between the magnetic source and the measurement point. $\mathbf{m}$ is the magnetic moment of the magnetic source. $\mathbf{n}=\mathbf{r} / r$ is the unit vector along $\mathbf{r}$.

The magnetic field vector at the point $(\mathbf{r}+\mathbf{n} d r)$ is:

$$
\mathbf{B}^{\prime}=\frac{\mu_{0}}{4 \pi}\left[\frac{3(\mathbf{m} \cdot \mathbf{n}) \mathbf{n}-\mathbf{m}}{(r+d r)^{3}}\right] .
$$

Then, the difference between $\mathbf{B}^{\prime}$ and $\mathbf{B}$ is:

$$
\mathbf{B}^{\prime}-\mathbf{B}=\frac{\mu_{0}}{4 \pi}[3(\mathbf{m} \cdot \mathbf{n}) \mathbf{n}-\mathbf{m}]\left(\frac{\partial}{\partial r} \frac{1}{r^{3}} d r\right)=-\frac{3}{r} \frac{\mu_{0}}{4 \pi} \frac{3(\mathbf{m} \cdot \mathbf{n}) \mathbf{n}-\mathbf{m}}{r^{3}} d r=-\frac{3}{r} \mathbf{B} d r .
$$

And

$$
\mathbf{B}^{\prime}-\mathbf{B}=\left[\begin{array}{l}
\nabla B_{x} \cdot \mathbf{n} d r \\
\nabla B_{y} \cdot \mathbf{n} d r \\
\nabla B_{z} \cdot \mathbf{n} d r
\end{array}\right]=\left[\begin{array}{lll}
\partial B_{x} / \partial x & \partial B_{x} / \partial y & \partial B_{x} / \partial z \\
\partial B_{y} / \partial x & \partial B_{y} / \partial y & \partial B_{y} / \partial z \\
\partial B_{z} / \partial x & \partial B_{z} / \partial y & \partial B_{z} / \partial z
\end{array}\right] \mathbf{n} d r=G \mathbf{n} d r
$$

According to Equations (4)-(6), the displacement vector is solved as:

$$
\mathbf{r}=-3 G^{-1} \mathbf{B}=-3 G^{-1}\left[\begin{array}{c}
B_{x} \\
B_{y} \\
B_{z}
\end{array}\right]
$$

\subsection{Localization Model}

For the MGT system, weak magnetic detectors with high precision, such as superconducting quantum magnetometer and fluxgate sensor, are usually used. Since the space of the casing is limited, the MGT system is composed of MEMS triaxial magnetometers. There are many kinds of MGT system, such as triangle, cruciform, tetrahedral, etc. $[15,16]$. Among them, the cruciform system meets the requirements of the narrow space of the casing. Moreover, compared with other systems, the cruciform 
system has better positioning accuracy and less systematic error, which can meet the requirements of the RHJD. In this paper, the localization method based on the cruciform system is studied.

Figure 2 shows the structure of the cruciform MGT system. There are 7 sensors in the same plane, of which N1 N5 are three-axis magnetometers, N6 is a three-axis accelerometer, and N7 is a three-axis gyroscope. The axes of each magnetometer are parallel to each other, and the baseline distance between adjacent magnetometers is $\mathrm{d}$. N1 can measure the magnetic field in the center of the sensor array, and it can also be combined with N6 to correct and compensate the attitude calculated by N7 to obtain the accurate attitude of the MGT system.

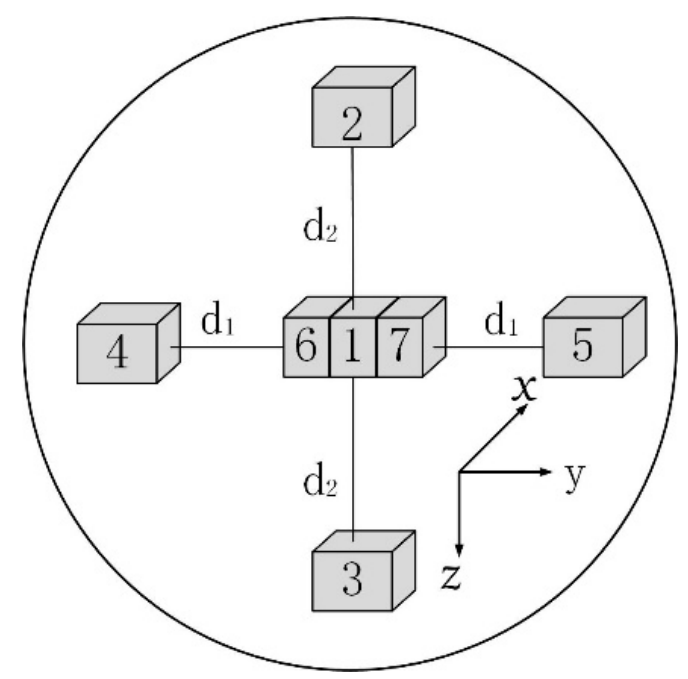

Figure 2. The structure of the cruciform MGT system.

Set the geomagnetic coordinate system $\mathrm{O}-\mathrm{XYZ}$ as the reference coordinate system, where $\mathrm{X}$ is northward, $Y$ is eastward, and $Z$ is vertically downward. The coordinate system of the MGT system is $\mathrm{O}-\mathrm{XYZ}$.

The effect of environmental magnetic fields, such as the geomagnetic field, on the localization algorithm is eliminated by means of difference calculation. At the beginning of the operation, the MGT system and the deflector are tripped down to the purposed depth, and the environmental magnetic field will be measured by the magnetometer $\mathrm{N} 1 \sim \mathrm{N} 5$ and recorded as $\left(B_{1 i x}, B_{1 i y}, B_{1 i z}\right), i=1,2,3,4,5$. Then the magnetizing nozzle is be tripped down, and the superposition value of the magnetic field of the magnetized nozzle and the environmental magnetic field at the measurement point will be measured and recorded as $\left(B_{2 i x}, B_{2 i y}, B_{2 i z}\right), i=1,2,3,4,5$. After the difference calculation of the two measurements, the magnetic field of the magnetized nozzle at the position of each magnetometer in the MGT system coordinate system can be obtained as follows,

$$
\left(B_{i x}^{\prime}, B_{i y}^{\prime}, B_{i z}^{\prime}\right)^{T}=\left(B_{2 i x}, B_{2 i y}, B_{2 i z}\right)^{T}-\left(B_{1 i x}, B_{1 i y}, B_{1 i z}\right)^{T}, i=1,2,3,4,5 .
$$

According to Euler theorem, the coordinate system of the MGT system can be obtained by the finite rotation of the geographic coordinate system. The angles between the three axes $x, y, z$ of the detection system and the coordinate axes of the geomagnetic coordinate system are $\alpha, \beta$ and $\gamma$. The rotation matrices $Q$ is [17]:

$$
Q=\left[\begin{array}{ccc}
1 & 0 & 0 \\
0 & \cos \alpha & \sin \alpha \\
0 & -\sin \alpha & \cos \alpha
\end{array}\right] \cdot\left[\begin{array}{ccc}
\cos \beta & 0 & -\sin \beta \\
0 & 1 & 0 \\
\sin \beta & 0 & \cos \beta
\end{array}\right] \cdot\left[\begin{array}{ccc}
\cos \gamma & \sin \gamma & 0 \\
-\sin \gamma & \cos \gamma & 0 \\
0 & 0 & 1
\end{array}\right]
$$


Thus, the magnetic field at the position of each magnetometer in the reference coordinate system $\mathrm{O}-\mathrm{XYZ}$ is:

$$
\left(B_{i x}, B_{i y}, B_{i z}\right)^{T}=Q^{T}\left(B_{i x}^{\prime}, B_{i y}^{\prime}, B_{i z}^{\prime}\right)^{T}, i=1,2,3,4,5 .
$$

The magnetic field at the center of the MGT system is measured by N1, the rate of change of the magnetic field on the $y$-axis is measured by $\mathrm{N} 2$ and N3, and the rate of change of the magnetic field on the $x$-axis is measured by N4 and N5. According to Equations (9) and (10), the difference calculation and coordinate conversion are carried out for the measured data. Then, the five independent components of the MGT can be obtained, and the Equation is:

$$
\left[\begin{array}{c}
B_{x y} \\
B_{x z} \\
B_{y y} \\
B_{y z} \\
B_{z z}
\end{array}\right] \approx\left[\begin{array}{c}
\Delta B_{x} / \Delta y \\
\Delta B_{x} / \Delta z \\
\Delta B_{y} / \Delta y \\
\Delta B_{y} / \Delta z \\
\Delta B_{z} / \Delta z
\end{array}\right]=\left[\begin{array}{l}
\left(B_{5 x}-B_{4 x}\right) / 2 d_{1} \\
\left(B_{3 x}-B_{2 x}\right) / 2 d_{2} \\
\left(B_{5 y}-B_{4 y}\right) / 2 d_{1} \\
\left(B_{3 y}-B_{2 y}\right) / 2 d_{2} \\
\left(B_{3 z}-B_{2 z}\right) / 2 d_{2}
\end{array}\right] .
$$

Combining Equations (9) and (10) obtains $\left(B_{1 x}, B_{1 y}, B_{1 z}\right)^{T}$, and then position can be calculated according to Equation (7),

$$
\mathbf{r}=-3 G^{-1} \mathbf{B}=-3 G^{-1}\left[\begin{array}{c}
B_{1 x} \\
B_{1 y} \\
B_{1 z}
\end{array}\right] .
$$

The vertical and lateral gradients of the geomagnetic field are generally about $20 \mathrm{nT} / \mathrm{km}$ and $5 \mathrm{nT} / \mathrm{km}$, respectively [18]. Compared with the magnetized nozzle, the MGT of geomagnetic field is extremely small. Moreover, for MGT systems with baseline distances less than $1 \mathrm{~m}$, the effect of the geomagnetic field can be neglected. In addition, the magnetic field data after the difference calculation can greatly eliminate the influence of the geomagnetic field and other interference magnetic field. Therefore, the influence of interference magnetic field on the MGT system can be ignored.

\section{Simulations}

According to the localization model, several simulations were carried out, and the applicability of the localization algorithm in RHJD was analyzed. The factors that affect the positioning accuracy are (without considering noise and system errors): (1) the distance between source (i.e., magnetized nozzle) and MGT system, (2) the strength of the equivalent magnetic moment of the source, (3) the accuracy of the magnetometer, (4) baseline distance.

Set the initial conditions of the localization model. The angles $\alpha, \beta$ and $\gamma$ are all equal to zero, the accuracy of the magnetometer is $1 \mathrm{pT}$, and the baseline distances $\mathrm{d}_{1}$ and $\mathrm{d}_{2}$ are equal to $0.1 \mathrm{~m}$. According to the drilling characteristics of RHJD, a survey line is selected (coordinate system of MGT system): 80 points are taken along the $x$-axis $(-40 \mathrm{~m} \sim 40 \mathrm{~m}), y=0 \mathrm{~m}, z=-1 \mathrm{~m}$, and equally spaced $(1 \mathrm{~m})$. The equivalent magnetic moment of the magnetized nozzle is $\left(M_{x}, M_{y}, M_{z}\right)=(10,1851,29) A \cdot m^{2}$.

\subsection{The Distance between Source and MGT System}

The simulation was carried out according to the initial conditions, and the results are shown in Figure 3. It can be observed that the localization method has a high accuracy, with the localization error less than $0.07 \mathrm{~m}$ and the maximum percentile error of $3.29 \%$. The maximum errors on the $x, y$ and $z$ axes are $0.06 \mathrm{~m}, 0.02 \mathrm{~m}$ and $0.05 \mathrm{~m}$, respectively. The errors on the $z$-axis are relatively large. The results show that the greater the distance between the detection system and the magnetized nozzle, the greater the localization error. Meanwhile, the localization algorithm can meet the requirements of drilling positioning for RHJD. 


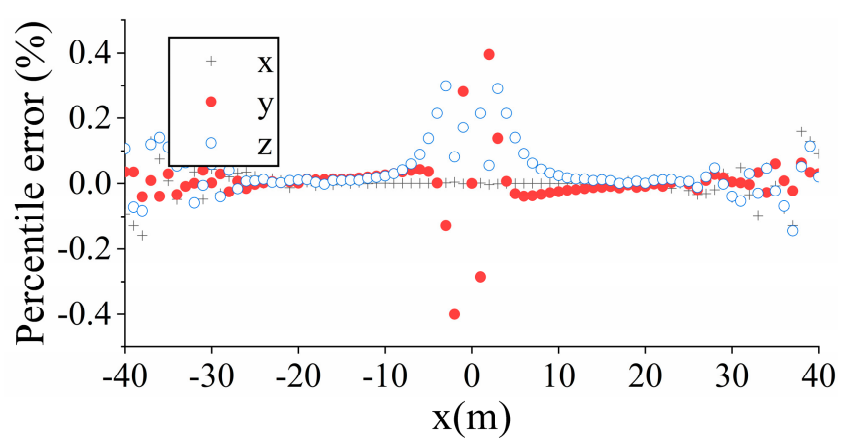

Figure 3. Localization errors varying with the distance between source and MGT system.

\subsection{The Strength of the Equivalent Magnetic Moment}

The equivalent magnetic moment was changed to $(50,9255,145) A \cdot m^{2}$, which is five times the original magnetic moment strength, and the others are consistent with the initial conditions. The results are shown in Figure 4. Compared with Figure 3, when the equivalent magnetic moment of the magnetized nozzle increases, the positioning accuracy is better and the maximum error is reduced to $0.03 \mathrm{~m}$. At a distance of $10 \mathrm{~m}$ to $40 \mathrm{~m}$, the percentile error is significantly reduced. Meanwhile, a variety of other changes were made, and a consistent conclusion was reached. Therefore, the equivalent magnetic moment can be improved by increasing the magnetization intensity of the nozzle, so as to reduce the localization error.

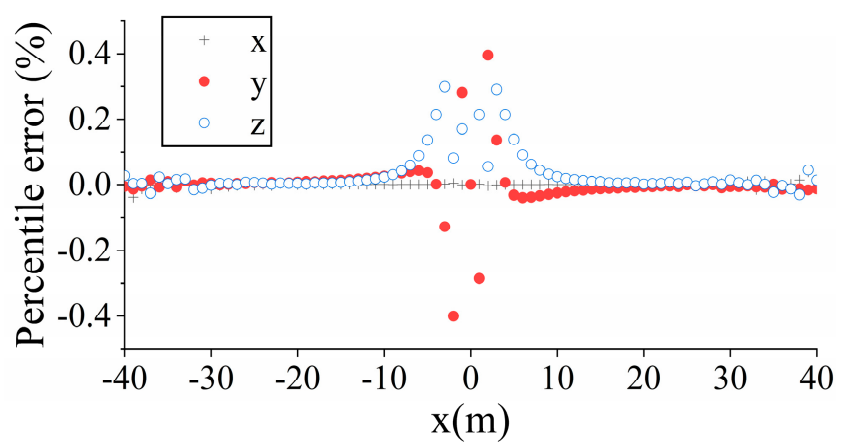

Figure 4. Localization errors varying with the distance between source and MGT system when the equivalent magnetic moment was changed to $(50,9255,145) A \cdot m^{2}$.

\subsection{The Accuracy of the Magnetometer}

The accuracy of the magnetometer was changed to $0.1 \mathrm{pT}$, and the others are consistent with the initial conditions. The simulation results are shown in Figure 5. Compared with Figure 3, it can be found that when the accuracy of magnetometers improves, the localization error significantly decreases. At a distance of $10 \mathrm{~m}$ to $40 \mathrm{~m}$, the percentile error is significantly reduced. The accuracy of magnetometers has great influence on the localization error.

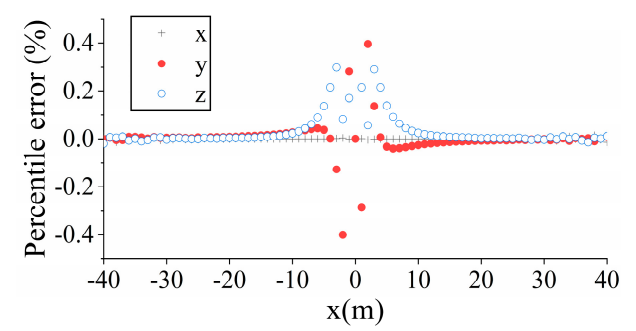

Figure 5. Localization errors varying with the distance between source and MGT system when the accuracy of the magnetometer was changed to $0.1 \mathrm{pT}$. 


\subsection{Baseline Distance}

Existing research on MGT-based localization method have all set $\mathrm{d}_{1}=\mathrm{d}_{2}$ in the discussion of baseline length, which is not comprehensive enough. In order to optimize the MGT system structure under the premise of meeting the engineering requirements of RHJD, a comprehensive analysis of the baseline distance is carried out. For RHJD, the baseline distance $d_{1}$ in the radial direction of the casing is limited due to the narrow space. However, the baseline distance $\mathrm{d}_{2}$ in the axial direction of the casing can be adjusted at will. According to the engineering practice, four points were taken along the $x$-axis $(5 \mathrm{~m} \sim 20 \mathrm{~m}), y=0 \mathrm{~m}, z=-1 \mathrm{~m}$, and equally spaced $(5 \mathrm{~m})$.

The localization errors at four measurement points were simulated by changing $\mathrm{d}_{1}$ from $0.05 \mathrm{~m}$ to $0.10 \mathrm{~m}$ with an interval of $0.005 \mathrm{~m}$, and $\mathrm{d}_{2}$ from $0.05 \mathrm{~m}$ to $0.55 \mathrm{~m}$, with an interval of $0.05 \mathrm{~m}$. The others are consistent with the initial conditions. In order to see the specific influence of the baseline distance on the positioning error more intuitively, absolute error is used here, and the results are shown in Figure 6.
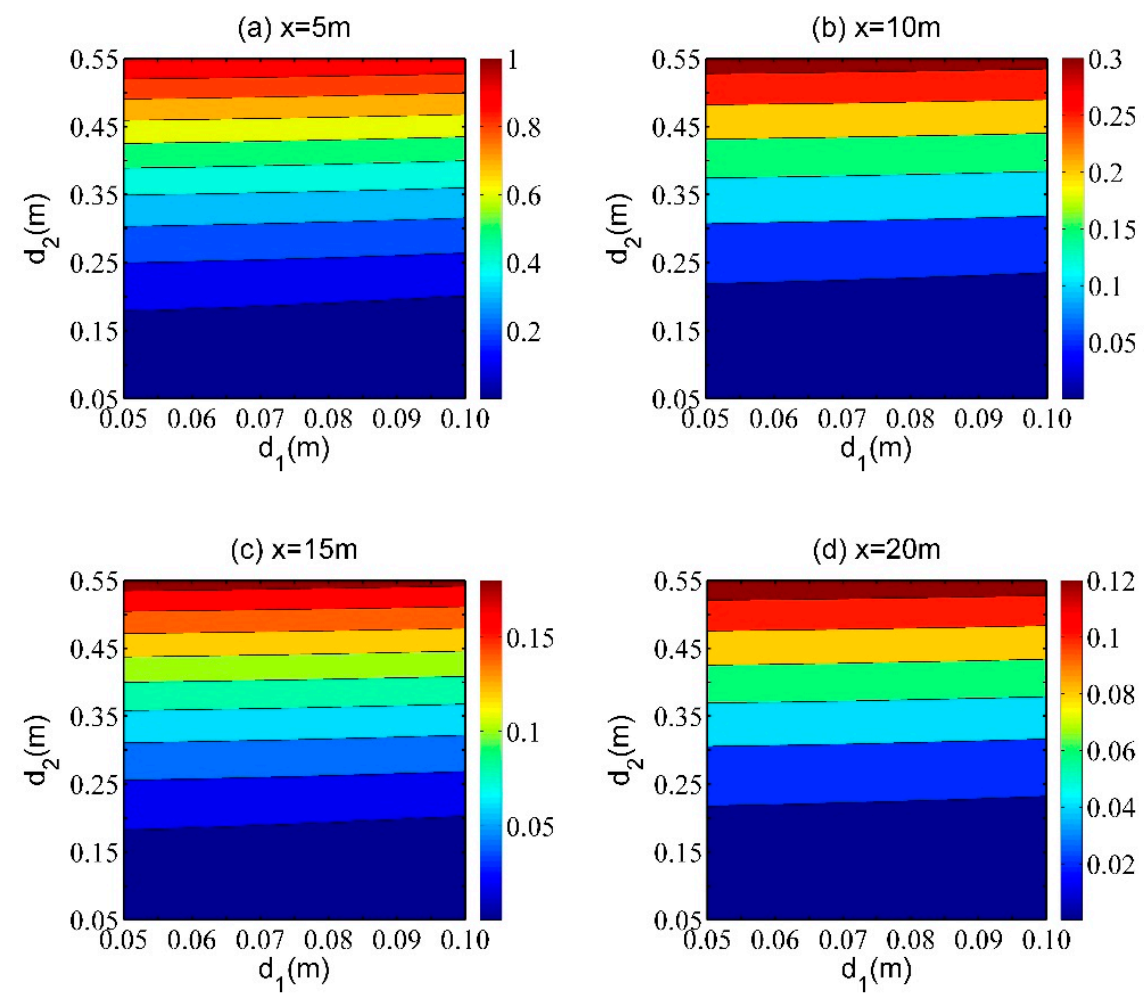

Figure 6. Localization errors varying with the baseline distances. (a) The localization errors varying with the baseline distances when the coordinates of the measuring point is $(x, y, z)=(5,0,-1) \mathrm{m}$. (b) The localization errors varying with the baseline distances when the coordinates of the measuring point is $(x, y, z)=(10,0,-1) \mathrm{m}$. (c) The localization errors varying with the baseline distances when the coordinates of the measuring point is $(x, y, z)=(15,0,-1) \mathrm{m}$. (d) The localization errors varying with the baseline distances when the coordinates of the measuring point is $(x, y, z)=(20,0,-1) \mathrm{m}$.

It can be seen that when the positioning distance is the same, the error increases with the baseline distance. When the baseline distance is the same, the error decreases with the increase in the positioning distance. This indicates that there is an optimal baseline distance within a certain distance to minimize localization error. The reason is that at a close range, reducing the baseline distance can improve the accuracy of the MGT information.

In summary, for the localization of magnetized nozzle based on MGT in RHJD, the distance between the magnetized nozzle and the MGT system, the strength of the equivalent magnetic moment of the magnetized nozzle, the accuracy of the magnetometer and the baseline distance have a great 
impact on the localization error. The positioning accuracy can be improved by reasonably selecting the accuracy of the magnetometer and the magnetization intensity of the magnetized nozzle and adjusting the baseline distance accordingly.

\section{Experiment}

In order to verify the practical effect of the localization method, a preliminary experiment was carried out using the MGT system composed of the $\mathrm{CH}-330$ digital vector magnetometer produced by $\mathrm{CH}-\mathrm{Hall}$ Electronic devices Inc. The range of the magnetometer is $0-100,000 \mathrm{nT}$ with an accuracy of $1 \mathrm{nT}$. Although the accuracy of the magnetometer used in the experiment is lower than that in the simulation, it can basically meet the requirements.

As shown in Figure 7, is the tri-axial fluxgate magnetometer and the MGT system. To ensure high consistency, we used the same magnetometer to measure the MGT. In order to eliminate measurement errors caused by zero offset and inconsistent sensitivity, which are common in vector magnetic sensors, we use the same three-axis fluxgate sensor to measure the magnetic field in the experiment, which improves the reliability and positioning accuracy of the experiment to a certain extent. There are sliding slots for the plastic hex bolts to slide freely and precise dimensioning lines on the aluminum disc. The movement and fixing of plastic bolts are used to control the position of the sensor, so that the MGT of the magnetic source can be measured by one sensor instead of five. The baseline distances were all $0.06 \mathrm{~m}$. The magnetized nozzle was replaced by a strong magnetic cylindrical permanent magnet with a diameter of $0.06 \mathrm{~m}$ and a length of $0.08 \mathrm{~m}$. By measuring the magnetic field in the space around the permanent magnet, its equivalent magnetic moment is $\left(M_{x}, M_{y}, M_{z}\right)=(681.76,0,0) A \cdot m^{2}$.

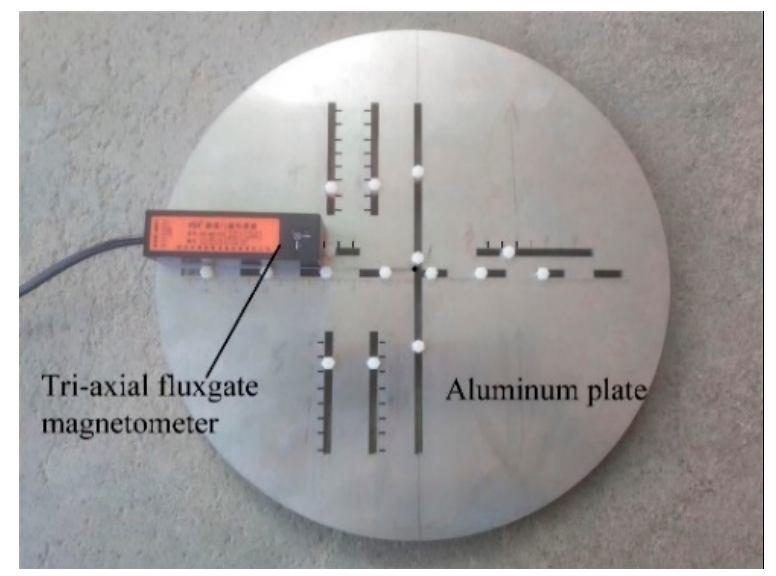

Figure 7. Tri-axial fluxgate magnetometer and magnetic gradient tensor system.

As shown in Figure 8, is the schematic diagram of the experiment. The MGT system was set up in the open area and its axes were coincident with the geomagnetic coordinate system. The orientation of the MGT system and the magnetic moment of the magnetic dipole have no effect on the positioning accuracy since the positioning is performed by measuring the MGT of the magnetic dipole (i.e., the magnetized nozzle). In the experiment, in order to facilitate the operation and simplify the calculation, the axis of the MGT system is coincident with the axis of the geomagnetic field. 


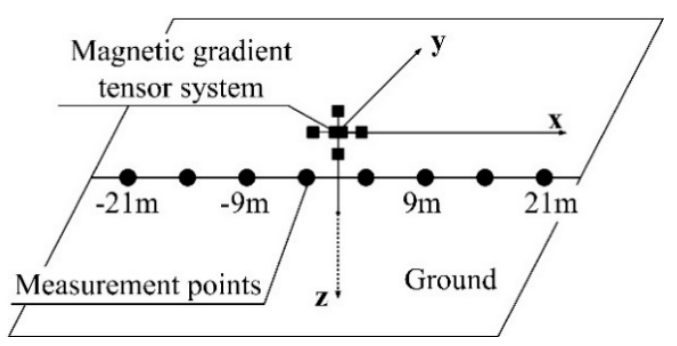

Figure 8. The schematic diagram of the experiment.

According to the engineering practice, six points were taken along the $x$-axis $(-21 \mathrm{~m} \sim 21 \mathrm{~m})$, $y=0 \mathrm{~m}, z=1 \mathrm{~m}$, and equally spaced $(6 \mathrm{~m})$. The permanent magnet was arranged at each point in turn, and the localization error obtained by using the localization method is shown in Figure 9.

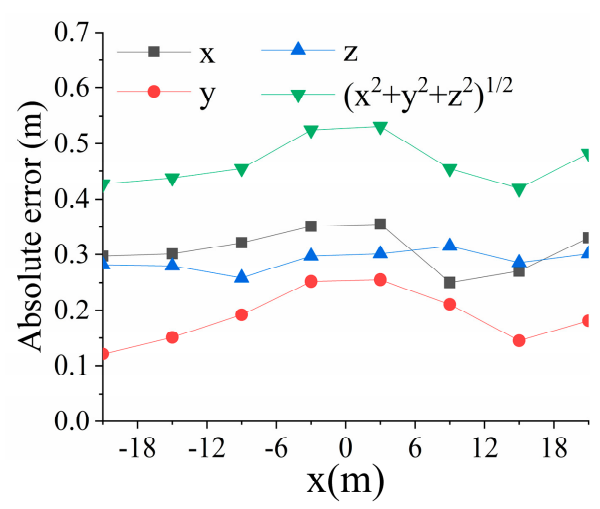

Figure 9. Localization errors in the experiment.

As can be seen from the Figure 9 that the localization error decrease with distance which is similar to the conclusion of simulation.

According to the conclusions of the simulation, the reasons for the larger error of the experiment compared with the simulation are that the magnetic moment of the magnet used in the experiment is smaller, and the accuracy of the magnetometer is lower. Meanwhile, the causes of errors may include magnetic interference of electronic equipment, orthogonality of sensor installation, no error compensation, etc. However, the preliminary experimental results show that the practical effect of the localization method meets the requirements of RHJD.

\section{Conclusions}

The difference calculation is used to improve the MGT-based localization method to eliminate the interfering magnetic field such as geomagnetic field, so that it can meet the engineering requirements of RHJD. Additionally, the MGT system is optimized according to RHJD technology.

Simulation results show that for RHJD technology, reducing the baseline distance, increasing the accuracy of the magnetometer and the intensity of the magnetic moment of the magnetic source can reduce the localization error. A preliminary experiment was carried out, and results similar to those from the simulation were obtained, which verified the correctness of the simulation. Meanwhile, the experimental results show that the positioning method has a good positioning accuracy within $20 \mathrm{~m}$, which can meet the requirements of RHJD.

The application of MGT-based localization method to RHJD not only expands the application field of localization method, but also makes theoretical innovation to RHJD.

Author Contributions: Conceptualization, M.W. and Z.L.; methodology, Z.L.; software, P.Z.; validation, M.W., and P.Z.; formal analysis, Z.L., R.X., T.L.; investigation, Z.L., Y.Z., C.Q.; resources, M.W.; data curation, M.W.; 
writing—original draft preparation, Z.L.; writing—review and editing, M.W. All authors have read and agreed to the published version of the manuscript.

Funding: This research was funded by State Key Laboratory of Petroleum Resources and Prospecting, China University of Petroleum, Beijing, grant number PRP/open-1610 and National Natural Science Foundation of China, grant number 51804267.

Conflicts of Interest: The authors declare no conflict of interest.

\section{References}

1. Chi, H.; Li, G.; Liao, H.; Tian, S.; Song, X. Effects of parameters of self-propelled multi-orifice nozzle on drilling capability of water jet drilling technology. Int. J. Rock Mech. Min. Sci. 2016, 86, 23-28. [CrossRef]

2. Bi, G.; Li, M.; Dou, L.; Qu, Z. Research on structure design and flow field characteristics of the novel jet bit for radial horizontal drilling. Energy Sci. Eng. 2018, 6, 535-547. [CrossRef]

3. Lu, Y.; Zhou, Z.; Ge, Z.; Zhang, X.; Li, Q. Research on and Design of a Self-Propelled Nozzle for the Tree-Type Drilling Technique in Underground Coal Mines. Energies 2015, 8, 14260-14271. [CrossRef]

4. Jacqueline, A.R.; Jefferson, D.M.P.; Joao, B.D.A.V.; Zhang, X.; Ricardo, C.B.D.M.; Luis, H.D.; Ricardo, S.A. New Cleanup System for Gravel-pack Completions: A Synergy of a Unique Acid System and Special Rotating Jetting Tool. In Proceedings of the SPE/ICoTA Coiled Tubing and Well Intervention Conference and Exhibition, The Woodlands, TX, USA, 20-21 March 2007.

5. Li, J.; Zhang, G.; Li, G.; Huang, Z.; Li, W. A method to double the extension ability of radial jet drilling technology. J. Energy Resour. Technol. 2018, 140, 093102.

6. Buset, P.; Riiber, M.; Eek, A. Jet Drilling Tool: Cost-effective Lateral Drilling Technology for Enhanced Oil Recovery. In Proceedings of the SPE/ICoTA Coiled Tubing Roundtable, Houston, TX, USA, 7-8 March 2001.

7. Dikken, B.J. Pressure Drop in Horizontal Wells and Its Effect on Production Performance. J. Pet. Technol. 1990, 42, 1426-1433. [CrossRef]

8. Beiki, M.; Clark, D.A.; Austin, J.; Foss, C. Estimating source location using normalized magnetic source strength calculated from magnetic gradient tensor data. Geophysics 2012, 77, J23-J37. [CrossRef]

9. Oruc, B. Location and depth estimation of point-dipole and line of dipoles using analytic signals of the magnetic gradient tensor and magnitude of vector components. J. Appl. Geophys. 2010, 70, 27-37. [CrossRef]

10. Sui, Y.; Miao, H.; Zhou, Z.; Luan, H.; Wang, Y. Correction and compensation of an airborne fluxgate magnetic tensor gradiometer. Explor. Geophys. 2017, 49, 726-734. [CrossRef]

11. Liu, L.; Gao, S.; Li, S.; Li, Z.; Xia, Y. Research progress of diffusion sensitive gradient field encoding schemes in magnetic resonance diffusion tensor imaging. Acta Phys. Sin. 2020, 69, 038702.

12. Wiegert, R.; Lee, K.; Oeschger, J. Improved magnetic STAR methods for real-time, point-by-point localization of unexploded ordnance and buried mines. In Oceans 2008; IEEE: Quebec City, QC, Canada, 2008.

13. Gang, Y.; Yingtang, Z.; Hongbo, F.; Zhining, L. Magnetic dipole localization based on magnetic gradient tensor data at a single point. J. Appl. Remote Sens. 2014, 8, 083596. [CrossRef]

14. Nara, T.; Suzuki, S.; Ando, S. A Closed-Form Formula for Magnetic Dipole Localization by Measurement of Its Magnetic Field and Spatial Gradients. IEEE Trans. Magn. 2006, 42, 3291-3293. [CrossRef]

15. Gang, Y.; Zhang, Y.; Fan, H.; Zhang, G.; Ren, G. Linear calibration method of magnetic gradient tensor system. Measurement 2014, 56, 8-18. [CrossRef]

16. Zhang, X.; Fan, L.; Cheng, P.; Chen, C.; Liu, X.; Kang, C. A Method to Remotely Track a Magnetic Target Using a Scalar Magnetometer Array. J. Sens. 2017, 2017, 6510980. [CrossRef]

17. Huang, X.; Chen, S.; Guo, S.; Xu, T.; Ma, Q.; Jin, S.; Chirikjian, G.S. A 3D Localization Approach for Subsea Pipelines Using a Spherical Detector. IEEE Sens. J. 2017, 17, 1828-1836. [CrossRef]

18. Lionel, M.; Pierre, N. Magnetic localization and identification of vehicles. In Unattended Ground Sensor Technologies and Applications; SPIE: Orlando, FL, USA, 2003. 